Slavica

bruxellensia

\section{Slavica bruxellensia}

Revue polyphonique de littérature, culture et histoire

slaves

12 | 2016

Miniatures

\title{
Le monde en miniature : le penzai littéraire de Radcliffe et son contexte polonais
}

\section{Anna Jaworska}

Translator. Dorota Walczak

\section{OpenEdition}

\section{Journals}

Electronic version

URL: http://journals.openedition.org/slavica/1752

DOI: 10.4000/slavica. 1752

ISSN: 2034-6395

\section{Publisher}

Université libre de Bruxelles - ULB

\section{Electronic reference}

Anna Jaworska, «Le monde en miniature : le penzai littéraire de Radcliffe et son contexte polonais », Slavica bruxellensia [Online], 12 | 2016, Online since 15 November 2016, connection on 03 May 2019 URL : http://journals.openedition.org/slavica/1752 ; DOI : 10.4000/slavica.1752

This text was automatically generated on 3 May 2019.

\section{(ब) $\Theta \Theta$}

Les contenus de Slavica bruxellensia sont mis à disposition selon les termes de la Licence Creative Commons Attribution - Pas d'Utilisation Commerciale - Pas de Modification 3.0 France. 


\title{
Le monde en miniature : le penzai littéraire de Radcliffe et son contexte polonais
}

\author{
Anna Jaworska \\ Translation : Dorota Walczak
}

La vie et l'œuvre d'Ann Radcliffe (1764-1823), auteure anglaise de romans gothiques, s'enferment dans de petits mondes tout comme la miniature d'un bateau est enfermée dans une bouteille de verre. C'est dans l'espace exigu de sa petite chambre que l'écrivaine créa les œuvres qui se sont inscrites dans les canons de la littérature anglaise. C'est une auteure importante pour l'espace slave et polonais en particulier. La traduction polonaise de son troisième roman The Romance of the Forest (La romance de la forêt, 1791), intitulée Puszcza czyli opactwo St.Clair (La forêt ou l'abbaye Saint-Clair), contient nombre de similitudes avec l'une des œuvres les plus importantes du romantisme polonais, le Konrad Wallenrod (Conrad Wallenrod, 1828) d'Adam Mickiewicz. L'auteure anglaise emploie des motifs similaires dans son roman (la vengeance, la trahison, le rêve, le suicide, l'orphelin, l'amitié, le chant, la tour, les fenêtres, les grilles, les lampes, le vent, la nuit, l'obscurité et la lumière).

En outre, sur la page de titre de la traduction polonaise figure une gravure avec des accessoires de la vignette accompagnant l'édition pétersbourgeoise du roman en vers de Mickiewicz mentionné plus haut, à savoir le panache de trois plumes que portaient sur leur casque les croisés, le manteau, l'épée associée au combat, ainsi qu'un instrument de musique évoquant le chant. L'hexamètre qui apparaît dans la phrase initiale de la traduction ainsi que la rupture du rythme de la phrase quand vient le mot ennemi comme si l'auteur de la traduction voulait attirer l'attention sur ce mot - constituent deux éléments indiquant aux lecteurs qu'ils ont affaire à une œuvre ressemblant à Konrad Wallenrod.

Les auteurs issus des pays slaves orientaux ont eux aussi été marqués par le roman de Radcliffe. Taras Ševčenko, auteur et poète ukrainien du XIX siècle, se réfère directement à cette œuvre dans son roman Muzykant (Le musicien, 1855) écrit en russe dans les 
dernières années d'exil. Le barde de l'Ukraine a décrit les alentours de la ville de Prylouky dans le gouvernement de Poltava, et le monastère qui s'y trouve. Mikhal Nikolaevič Zagoskin, dans son roman Iskusitel (Le tentateur, 1838) évoque également le troisième livre de l'auteure dans le dialogue de ses héros ${ }^{1}$. Comment donc est née cette œuvre qui a fasciné les Slaves?

Radcliffe faisait partie de ses auteurs dont les longues heures passées dans la solitude étaient entièrement consacrées à l'écriture. Elle n'a pas écrit de mémoires ni beaucoup de lettres. Une telle attitude était difficilement acceptable par les cercles littéraires des écrivains et des lecteurs d'antan. Ils avaient du mal à admettre une telle ignorance au sujet de l'auteure la plus connue d'Angleterre, voire d'Europe. C'était le prix à payer que Marguerite Duras souligne ainsi dans l'un de ses récits : «Il faut toujours une séparation d'avec les autres gens autour de la personne qui écrit les livres. C'est une solitude essentielle. C'est la solitude de l'auteur, celle de l'écrit. [...] Cette solitude réelle du corps devient celle, inviolable, de l'écrit. $»^{2}$

En 1791 parait le troisième roman de l'écrivaine intitulé The Romance of the Forest. Bien qu'il soit plus tardif que ses deux livres précédents - The Mystries of Udolpho (Les Mystères du château d'Udolphe, 1794) et The Italian, or the Confessional of Black Penitents (L'Italien ou le Confessionnal des pénitents noirs, 1797) - c'est cette publication qui a rendu célèbre le nom de Radcliffe. Cette œuvre a été accueillie avec beaucoup plus d'enthousiasme que ses livres postérieurs.

Jusqu'à la fin du XVIII siècle, six éditions du roman ont été publiées par la maison d'édition de Thomas Hookham et James Carpenter. Peu de temps après ont paru des traductions en diverses langues européennes. Le livre est paru en allemand en 1792, deux ans après l'édition originale; il paraît en français en 1794. La traduction russe a été réalisée dans les années 1801-1802. Pour la traduction polonaise, il a fallu attendre trentehuit ans après la parution originale. C'est en effet en 1829, qu'a vu le jour Puszcza czyli opactwo St. Clair, la traduction The Romance of the Forest, dans l'imprimerie de Józef WĘcki, célèbre libraire et imprimeur varsovien. Aujourd'hui, cette traduction est totalement oubliée. Puszcza czyli opactwo St. Clair a été non seulement le premier livre d'Ann Radcliff traduit en polonais mais aussi le premier roman gothique anglais introduit dans le champ littéraire polonais. Seuls deux exemplaires complets des quatre volumes de la traduction polonaise existent aujourd'hui. Après avoir fait des recherches dans nombre de bibliothèques polonaises et étrangères (en particulier russes), j'ai retrouvé un de ces exemplaires en février 2013 dans le catalogue de la Bibliothèque Universitaire de Varsovie sous la côte 17.6.2.7a, 17.6.2.7b, 17.6.2.7c, 17.6.2.7d. Un deuxième exemplaire est conservé à la Bibliothèque Jagellonne de Cracovie. Il s'est probablement retrouvé dans cette bibliothèque après la liquidation d'une librairie cracovienne ou d'une bibliothèque, appartenant vraisemblablement à Józef Czech ou à Edward Friedlein. Il a également pu être donné par le libraire varsovien Gustaw August Sennewald, qui a offert à la Bibliothèque Jagellonne une partie de ses livres, parmi lesquels se trouvait entre autres Puszcza czyli opactwo St. Clair. Soulignons que chacun des quatre tomes de la traduction constitue un petit format que l'on pourrait appeler de poche.

J'interrogerai dans cet article le roman de Radcliffe et sa traduction de petit format; je montrerai comment l'auteure y mit, comme dans une boule de verre, des miniatures de personnages qui lui sont contemporains (par exemple les membres de la lignée des SaintClair, liés aux terres polonaises) ainsi qu'un petit panorama de la situation mondiale de l'époque (en particulier en France). La miniature de la pensée révolutionnaire de l'époque 
contenue dans le roman pouvait être du goût des Polonais à une époque où la Pologne n'existait plus sur les cartes géographiques : ils pouvaient y retrouver une miniature de Konrad Wallenrod et des motifs de la lutte populaire illégale au XIX ${ }^{\mathrm{e}}$ siècle.

Les circonstances de l'édition de cette traduction cachent beaucoup de mystères: par exemple nous ne trouvons pas de nom de traducteur sur la couverture. Nous pouvons pourtant deviner que l'auteur en fut Stanisław Żugarzewski, un insurgé de 1830 originaire de la région de Vilnius qui, après les représailles de l'Insurrection de Novembre, a émigré et s'est établi en France. S'il est réellement le traducteur de Puszcza, son destin l'a projeté dans le lieu où s'est déroulée l'action du roman qu'il a traduit. La traduction polonaise de The Romance of the Forest n'est pas une traduction de l'anglais, mais du français - La forêt, ou l'Abbaye de Saint-Clair ayant été traduit par François Soulès ${ }^{3}$ sur la base de l'original anglais ${ }^{4}$.

Dans ce troisième roman, Radcliffe invite les lecteurs à visiter le microcosme créé par la culture française. L'auteure s'est probablement inspirée de personnages français réels. Il est intéressant de noter que certains d'entre eux sont également liés aux terres polonaises. De cette façon, quelques tomes du roman contiennent des miniatures de personnages réels. Le héros Pierre de la Motte avait des homonymes dans le monde réel. Le premier d'entre eux, Pierre de la Motte (décédé en 1685), était capitaine de la compagnie du Régiment de Carignan-Salières envoyée en Nouvelle France (c'est-à-dire au Canada) par le roi Louis XIV pour protéger les colons français des Iroquois. Le second, Pierre Lambert de La Motte (1624-1679), était un évêque français, co-fondateurs de la Mission Etrangère à Paris. Le nom de la Motte pouvait également évoquer l'affaire du collier de la reine qui a fait grand bruit dans l'opinion publique de l'époque. Les acteurs de ce scandale étaient Jeanne de Valois-Saint-Rémy, comtesse de la Motte (1756-1791), qui prétendait être une proche de la reine Marie-Antoinette, et Louis René Édouard de Rohan (1734-1803). Enfin, le comte Toussaint-Guillaume Picquet de La Motte (1720-1791) était un amiral français.

L'année de parution de l'original anglais a également été marquée par des événements qui étaient lié au roman de Radcliffe. A l'époque, sont morts Jeanne de la Motte et le comte amiral Picquet de la Motte, John Sinclair publia le premier tome de Statistical Accounts of Scotland, et outre-atlantique eut lieu la bataille de Wabash sous le commandement d'Arthur de Saint-Clair, et dont les membres non seulement protégeaient l'abbaye de Saint-Clair présente dans le roman de Radcliffe, mais se sont également inscrits dans l'histoire de la Pologne. La présence de membres de cette illustre lignée sur les terres polonaises remonte au mariage de l'aristocrate écossais Alexandre Bower de Saint-Clair, ayant servi dans la marine militaire indienne, avec Pelagia Kossakowska, fille de Józef Dominik Kossakowski, amie de longue date du prince Adam Czartoryski.

Leur arrière-petit-fils Stanisław Saint-Clair a participé à l'insurrection de Janvier (1864), et a vécu après chez son frère Alexandre à Jassy. En mai 1877, il essaya d'organiser une légion polonaise auprès de l'armée turque. Un an plus tard, au début du printemps 1878, il a été reçu par les autorités turques pour proposer un plan d'insurrection dans les Rhodopes. Il avait l'intention de traverser le Danube à la tête de sections d'insurgés, d'entrer sur les terres polonaises et de rallumer l'élan national antirusse. Ces plans n'ont toutefois pas été réalisés et ont vite été abandonnés. En 1879, il s'est installé en Belgique. Il est mort le 27 janvier à Ligneuville. Il repose dans le cimetière local. Son histoire, telle une boule de verre, contient les rêves d'indépendance et d'autonomie du XIX ${ }^{e}$ siècle polonais. 
Notons que le roman de Radcliffe est fidèle à l'histoire : l'action se déroule dans la France $\mathrm{du} \mathrm{XVII}^{\mathrm{e}}$ siècle. Le voyage du lecteur est ainsi ponctué par des cas extraordinaires et de brusques changements du cours des événements. Voici Pierre de La Motte avec sa femme Konstancja ${ }^{5}$ (Constance) de La Motte et leurs servants fuyant sous la protection de la nuit bruyante de Paris. Pendant leur long voyage, ils se livrent à des réflexions sur leur passé douloureux. On apprend que les soucis financiers sont la cause de leur départ soudain de la capitale, la peur de créanciers exigeant le règlement de leurs dettes.

Leur carrosse traverse une forêt pendant qu'un violent orage s'abat sur eux. Monsieur La Motte suit la petite lumière vacillante d'une bougie à la fenêtre d'une maisonnette aperçue entre les arbres. Il décide de quitter la voiture et de demander aux habitants de la maison le chemin vers la ville la plus proche. La Motte, dirigé par sa curiosité, passe le seuil de la maison et est aussitôt poussé à l'intérieur où est amenée une fille en pleurs. Un grand personnage masqué permet à La Motte de quitter les lieux à la condition de prendre avec lui la jeune femme; il ordonne également de ne jamais revenir dans ces contrées. En conséquence de l'accord de La Motte, les héros quittent la France.

$\mathrm{Au}$ début, la fille pensive et tremblante ne veut pas parler d'elle. Mais au fur et à mesure du chemin parcouru, elle prend confiance et raconte à Madame La motte, si bienveillante à son égard, l'histoire de sa vie. Elle a été, après la mort de sa mère, confiée à un cloître, mais a refusé de prononcer ses vœux et a ainsi provoqué la colère de son tuteur qui, dans un acte de vengeance, l'a emmenée dans un chalet situé au milieu d'une forêt et l'a livrée aux mains d'hommes douteux. Et c'est justement à la porte de ce chalet caché que Monsieur La Motte avait frappé.

Après des jours et des jours de voyage, tous entrent finalement dans une grande forêt située au nord de la France. Le crépuscule tombe. L'une des roues de la diligence se brise, continuer le voyage s'avère impossible. Les protagonistes décident de loger dans les murs d'une abbaye abandonnée à côté de laquelle ils sont passés auparavant. La famille découvre dans la chapelle une quantité de pièces qui ont été ajoutées plus tard, et décide d'y loger.

Depuis ce moment la forêt - loin de la civilisation - devient avec l'abbaye un monde en miniature, un monde à part, autonome et enfermé dans les pages de ce roman.

Radcliffe, véritable maître du penzai ou pensai (penjing) - rappelons qu'il s'agit au départ d'un terme qui dans la langue et culture chinoises désigne un arbre unique dans une coupe -crée un monde en réduction avec une virtuosité étonnante. Au centre de ce monde, l'auteure place une bâtisse gothique resplendissante. Il y a des similitudes entre la construction des cathédrales et la composition de penzai. Dans les deux cas, il n'y a pas de place pour le hasard et le moindre élément insignifiant est choisi avec soin.

La construction de l'œuvre de Radcliffe est très organisée, c'est pourquoi il est possible de la comparer aux cathédrales gothiques ou aux penzais où le hasard n'a pas sa place. Les citations d'autres auteurs et la devise de la tragédie Macbeth, en apparence peu significatives, en constituent un exemple parfait. Ils n'ont pas seulement une fonction ornementale, mais jouent un rôle clé dans l'interprétation. La romancière entre également en dialogue avec la tradition de la littérature européenne, s'inspirant d'œuvres anglaises, italiennes, françaises et de la mythologie. Les nombreuses œuvres poétiques citées dans le texte original, malheureusement non traduites par le traducteur polonais, jouent également un rôle important: elles créent une atmosphère particulière et caractérisent le personnage principal, la poétique Adeline. 
Je compare l'œuvre de Radcliffe au penzai parce que ce dernier sedistingue des autres arts de créer des paysages comme le sakei, par exemple- l'art de créer les paysages en miniature et des figurines en miniature qui y sont placées. L'écrivaine anglaise (et la traduction polonaise le souligne) fait aussi en sorte que ses "petits » personnages, dans l'espace restreint de la forêt et de l'abbaye soient des héros aux teintes et couleurs différentes qui se meuvent et agissent.

\section{Les lois du microcosme}

Radcliffe crée un monde littéraire en miniature qui a ses propres lois et qui échappe à toute classification uniforme. Dans The Romance of the Forest l'action passe par des méandres et des détours improbables comme dans le célèbre roman de Fiodor Dostoïevski (1821-1881), Crime et Châtiment (ПРЕСТУПЛЕНИЕ И НАКАЗАНИЕ, 1866). Ainsi, tout d'abord la roue de la voiture casse et les héros sont obligés de chercher abri dans les vestiges de l'abbaye. Plus tard, quand Adeline essaye de fuir, elle sera capturée par le marquis et placée à nouveau dans la bâtisse gothique. Le lecteur de ce roman peut avoir l'impression qu'une force mystérieuse l'empêche de s'éloigner du lieu où se trouve le squelette de son père. Il vaut la peine de remarquer que la plus grande partie de l'action se déroule dans les ruines habitées autrefois par des moines et non dans le château qui a été le lieu de l'action des romans précédents. Ainsi, dans ce roman règne une ambiance mystique, et l'abbaye devient un axis mundi littéraire où les trois sphères, à savoir la terre, le ciel et l'enfer du cosmos se rejoignent. Dans ce lieu, la rupture de l'unité de l'espace est interrompue et le passage d'une région du cosmos à l'autre est rendu possible ${ }^{6}$.

L'étymologie du mot abbaye en français ou abbey en anglais est intéressante. Le mot latin abbas provient du mot araméen אבא (abbā) qui signifie « le père » 7 . Il peut s'agir à la fois du père terrestre ou du Créateur, le Père, et Opatrzność - la Providence (le traducteur utilise ce mot-là ${ }^{8}$ ). De plus, le prénom du sauveur d'Adeline - Théodore - provient du

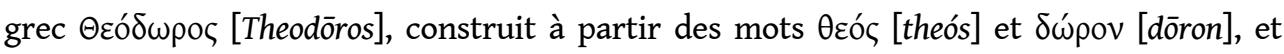
signifie « le don de Dieu » ou «donné par Dieu »". Difficile donc de ne pas associer la Bible à la lecture du roman. La scène finale à Paris ressemble fort au Jugement Dernier qui mettra fin à toutes sortes de méfaits. Le motif du meurtre de son frère fait allusion à l'histoire d'Abel et Caïn du Livre de la Genèse. Dans les rêves d'Adeline, un homme d'une trentaine d'années est couché sur le lit et à certain moment, le sang coule de son flanc comme du corps du Christ transpercé sur sa croix par la lance à l'âge de trente-trois ans. Dans ce rêve, les personnes pleurant le mort se trouvent dans la chambre d'Adeline. La vision qui y est présentée rappelle le tableau Cristo morto (Le Christ Mort, environ 1480) du peintre italien Andrea Mantegna. Dans la traduction polonaise, sa ressemblance avec l'univers de cette petite chambre est aussi soulignée par la traduction apparentée au style de phrases utilisées par Jakub Wujek dans sa traduction de la Bible (1559). Ce contexte est essentiel pour un lecteur polonais car la traduction de Wujek qui a été la référence pendant presque 300 ans (jusqu'en 1965, quand est parue la Biblia TysiĄclecia, la «Bible du millénaire ») a formé la langue polonaise, et influencé nombre d'œuvres littéraires, entre autres Ksizgi narodu polskiego i pielgrzymstwa polskiego (Les Livres de la nation polonaise et du pèlerinage polonais, 1832) de Mickiewicz.

Bâties dans un style gothique ${ }^{10}$, les abbayes renouent avec la symbolique des églises du moyen-âge. Rappelons que les bâtisseurs de cathédrales, tout comme les platoniciens, accordaient une importance immense aux mathématiques et à la géométrie, puisqu'elles 
sont les intermédiaires dans les relations entre le divin et l'humain. Les chiffres ont été significatifs dans l'architecture gothique, mais aussi dans les croyances des différents peuples, dans la Bible, ainsi que dans la Cabale. Cette dernière aidait ses interprètes à résoudre les différents versets. Les chiffres dans le texte de Radcliffe prennent aussi une couleur particulière puisque l'héroïne voit l'avenir dans trois rêves qui se succèdent. Ce chiffre trois symbolise la victoire sur la division, l'ensemble du début et de la fin, le passé et le présent ainsi que l'unité de la famille qui se compose de deux parents et d'un enfant ${ }^{11}$. L'apparition du fantôme de la fille dans ces trois visions oniriques est donc très significative.

Soulignons que les motifs qui apparaissent dans le microcosme de The Romance of the Forest, que l'on peut comparer aux éléments de construction de cathédrales gothiques, n'ont pas été utilisés par hasard; ils s'inscrivent dans la longue tradition des rêves bibliques. Selon la Bible, Dieu a été actif pendant que l'homme dormait dans un sommeil profond. Rappelons qu'Adeline rencontre un paon dans les ruines de l'abbaye. Cela semble extraordinaire, car cet oiseau était associé aux jardins des palais et de la cour. Sa présence n'est ainsi pas dépourvue de sens et semble être un symbole de la royauté ${ }^{12}$, de la résurrection et de la vie éternelle ${ }^{13}$. A l'époque, on croyait en effet que les âmes des morts revenaient sur terre sous forme d'oiseaux (des hiboux et des corbeaux peuplent également le roman). Le paon est toutefois aussi l'attribut de la déesse grecque Hera, la patronne des mariages et des femmes mariées, et sa queue multicolore rappelle également les vitraux des temples gothiques.

D'autres accessoires tels que la roue cassée sont eux aussi enracinés dans la symbolique mystique et religieuse. Le Grand Architecte de l'Univers qui se sert d'un compas représenté sur les pages-cartes de l'œuvre de Radcliffe, nous est connu d'un tableau de William Blake, The Ancient of Days (L'ancien des Jours), ainsi que des visions du prophète Ezéchiel dans lesquels les Pères de l'Eglise voient un rapport avec les quatre éléments et les quatre saisons ${ }^{14}$. Pour Hildegarde Von Bingen, femme savante et mystique, la roue était le symbole de la divinité. Quand la roue tourne, nous pouvons aussi la comprendre comme un symbole de la vie, de son instabilité et de ses changements. Par ailleurs, Tyché, la déesse grecque du destin, est présentée sur une roue car celle-ci représente les jugements des tribunaux. Enfin Némésis, la déesse de la vengeance, figure souvent sur une roue qui est aussi un des plus anciens attributs solaires.

La racine - celle qui brise la roue dans la forêt où voyagent les protagonistes - constitue l'une des parties les plus importantes d'une plante, elle signifie le début et la source invisible de ce qui existe ${ }^{15}$. Dans de nombreux textes sacrés, notamment les Upanishad, les racines de l'arbre du monde sont dirigées vers le haut, et sa frondaison pend vers la terre. Une vision semblable apparaît chez Dante Alighieri dans la Divina Commedia, où les sphères célestes de l'au-delà créent une figure en forme d'arbre retourné - les racines vers le haut. Dans la Bible, on peut rencontrer beaucoup d'images également liées aux racines.

Ainsi l'orage, la nuit, la pluie et le vent ${ }^{16}$ appartiennent à l'univers du roman métaphysique, du roman mystique et du roman gothique, nous retrouvons en outre leurs caractéristiques chez de nombreux auteurs anciens ${ }^{17}$. Est-ce réellement par hasard que l'héroïne découvre la chambre dans laquelle son père a été tué, sachant que c'est un morceau de tissu s'agitant sous le vent qui lui a dévoilé son entrée? La présence de paysages nocturnes dans les pages de The Romance of the Forest est également importante. Elle indique l'importance suprême de la nuit opaque avant la venue du soleil et de la lune, 
elle est l'espace temporaire permettant de contacter les puissances occultes qui aident l'homme à examiner l'avenir ${ }^{18}$. En même temps, la nuit permet de se libérer des détails tels que les chiffres, les couleurs, les formes ${ }^{19}$, tandis que les coups de tonnerre qui retentissent dans la nuit rappelent aux héros les pouvoirs divins qui suggèrent alors l'ordre potentiel dans le microcosme de la nuit terrestre ${ }^{20}$.

Remarquons que l'auteur de la traduction polonaise, tout comme Radcliffe dans l'original, se réfère au facteur transcendant, il le nomme: moc nadnaturalna («puissance surnaturelle ») $)^{21}$, Najwyższa Istota ( ( Être Suprême ») ${ }^{22}$, Wielki Stwórca Natury (Grand Auteur de la Nature) $)^{23}$, mĄdrość wyższa nad ludzką (au-dessus de la sagesse humaine), Przedwieczny (Fils de la Justice) ${ }^{24}$ ou Bóg (Dieu) ${ }^{25}$. L'expression «Être suprême » mérite une attention particulière. Cette mention était populaire en France pendant la révolution, époque de la première traduction de The Romance of the Forest dans la langue de Molière. Cela a pu également servir à augmenter l'intérêt des Français pour ce livre qui promeut plutôt une vision déiste. Dans le monde de Radcliffe, les forces surnaturelles interviennent dans le destin de l'homme : « ie ciel vous envoie à mon secours $»^{26}$, « il semble que sa conservation est l'ouvrage de quelque chose au-dessus de la sagesse humaine $»^{27}$, « les circonstances qui avaient accompagné la découverte de ces papiers ne lui parurent plus avoir été l'effet du hasard, mais celui d'une Puissance surnaturelle dont les desseins sont grands et justes $»^{28}$.

Après une analyse approfondie du texte et de son contexte, nous nous rendons compte que les dates qui apparaissent dans le roman ne sont pas fortuites. La Motte écrit sur la porte de l'abbaye qu'il y est venu le 27 avril 1656. Or, il s'avère que c'était un jeudi, onze jours après Pâques (qui était alors tombé le 16 avril 1656), lorsque les onze apôtres restent à Jérusalem après la trahison de Judas (Actes des Apôtres, I, 26). Par ailleurs, Pâques nous renvoie à la promesse d'une nouvelle vie.

Le mois d'octobre - mois de la capture du père d'Adeline - apparaît lui aussi chargé de signification. C'est le temps des vendanges, ce qui nous envoie à la symbolique des vendanges du Jugement Dernier (Apocalypse 14, 14-20). Le 12 octobre 1642 était de plus le quatrième jour de la fête juive de Sukkot (סוכות), aussi appelée en polonais la Fête des Tentes (ŚwiĘto Namiotów), qui tombe durant le mois du tichri (תשרי) commémorant le séjour des Hébreux dans le désert, sous des tentes, pendant leur cheminement vers la terre promise de Canaan. En 1642, le 12 octobre est aussi tombé un dimanche, jour lié à la symbolique de la résurrection et du renouveau. En outre, selon les règles de la numérologie, après avoir fait l'inventaire de tous les chiffres, nous obtenons le résultat [1 $+2+1+0+1+6+4+2=17 ; 1+7=8]^{29}$, soit le chiffre 8 qui occupe un rang supérieur dans la Bible : huit personnes se trouvent dans l'Arche de Noé (Genèse 6,18$)$, il y a huit bénédictions (Matthieu 5, 3-10), et selon les calculs, dans la tradition judaïque, Jésus a ressuscité le huitième jour. Après les sept jours de la semaine, le huitième arrive : le début d'un temps nouveau, sans souffrance ni mort. Le nom même de Jésus est lié au chiffre 8, selon l'art de la gématrie (c'est-à-dire la transformation des mots sémites et grecs en chiffres): le nombre 888 est la somme des lettres grecques Ihsous (Jésus) - I (10) H (8) S (200) O (70) U (400) S (200). Le prénom du père d'Adeline, Henri, commence par la lettre $H$ à laquelle on attribue le nombre 8 , et dans le huitième chapitre (du deuxième volume) au point culminant du roman, l'héroïne retrouve la chambre dans laquelle a été tué son père. Ajoutons pour finir que la somme des chiffres des numéros de chapitres de The Romance of the Forest dans l'original anglais (26) égale à $8(2+6)$, soit les mêmes chiffres que la date du 26 avril (de nouveau 2+6), date à laquelle a été ordonnée l'exécution d'Adeline. 
Il se trouve que l'écrivaine Mary Edgeworth, dans sa lettre du 14 août 1792 à sa cousine Sophy Ruxton de Clifton, écrit que le fait que ce livre a quelque chose de The Castle of Otranto (Le Château d'Otrante, histoire gothique, 1764) ${ }^{30}$ ne serait pas dû au hasard. En effet, dans cette œuvre de Horace Walpole, tout comme dans The Romance of the Forest, apparait l'horreur métaphysique et sacrale. Le héros est ici un Dieu sévère et juste, et le sort d'Adeline doit montrer son pouvoir sur le réel à la déesse de la divine Providence.

\section{Dans la forêt : d'un symbole au symbolisme universel}

Autour du château de Manfred, comme probablement autour de l'abbaye de Saint-Clair se trouve une forêt dense, impénétrable - à la fois lieu sombre et grave, auquel s'ajoute une riche symbolique. Il est clair que la forêt a joué un rôle créateur et culturel pendant des siècles, qu'elle a inspiré tout un imaginaire. ${ }^{31}$ On retrouve sa symbolique dans la Divina Commedia de Dante; dans Macbeth également, nous retrouvons la présence de la forêt de Birnam dans les prophéties des sorcières. De même, le crime du Marquis de Montalt n'est pas révélé jusqu'au moment où Pierre de la Motte et Adeline affrontent les profondeurs de la forêt. Dans l'œuvre de Shakespeare la nature s'oppose aux crimes atroces ; la nuit de la mort de Duncan, des anomalies météorologiques ont lieu, les animaux sont inquiets. Dans The Romance of the Forest, la nature intervient également dans le destin des héros en enfermant ceux-ci dans un cercle clos. La notion de cosmos enfermé a été déjà introduite par John Milton dans Comus (1634): "Dans les bois et les lugubres enchantements", quand Rictor Norton annonce ainsi que "dans chaque siècle doivent se cacher des horreurs séduisantes $\rrbracket^{32}$. Rappelons également que les arbres, dans l'interprétation culturelle, signifient la croissance symbolique et spirituelle, puisqu'ils nous renvoient au «je» microscopique mais qui devient grand, puisqu'il est compris comme part universelle du macrocosme vers lequel nous nous dirigeons toute notre vie ${ }^{33}$. Cette symbolique correspond parfaitement à la présence du facteur métaphysique que nous avons traité dans la partie précédente.

Norton avance une hypothèse dans laquelle chez Radcliffe la forêt symbolise les aberrations créatives d'un cerveau malade qui démolit et crée une nouvelle structure de la société et de la civilisation ${ }^{34}$. Le voyage dans les ténèbres de la forêt ressemble au chemin que doit traverser un poète pour découvrir ses propres puissances créatrices. Et pour Adeline, âme sensible, c'est un lieu sacré car de la Motte traite ces éléments de la nature d'une manière pragmatique, comme un abri où il peut se cacher de ses créanciers. C'est à l'opposé du ressenti d'Adeline qui est capable, via ses sentiments, d'augmenter les dimensions du microcosme du lieu concret au macrocosme du ressenti: "Adeline jusqu'alors muette, jeta un cri mêlé d'admiration et de crainte. Une sorte d'effroi délicieux s'emparait de son âme et faisait palpiter son sein $»^{35}$.

Rappelons que dans la littérature polonaise le premier texte où le motif de la nature apparaît d'une manière distincte est un écrit en latin, Carmen de bisontis ${ }^{36}$ (1523), de la plume de Mikołaj Hussowski, poète polono-latin du début de la Renaissance polonaise. Nous pouvons y repérer le caractère spécifique d'un paysage polonais particulièrement en contraste avec le paysage méditerranéen si populaire à l'époque de la Renaissance. Comme le remarque Tadeusz Komendant, notre civilisation a commencé dans le jardin, au sud de l'Europe ${ }^{37}$. Les barbares venaient du dehors des frontières, du Nord, des forêts et avec eux, les Goths. Ce n'est pas par hasard si l'adjectif « gothique » a souvent été compris et utilisé en tant que synonyme de «barbare $\aleph^{38}$. Si les auteurs de la vieille Pologne - 
Hussowski mis à part - se focalisaient sur la poésie et les forêts utiles, les romantiques plaidaient quant à eux pour les forêts vierges et intactes du Nord ${ }^{39}$. La forêt est ainsi un élément mystique important dans Genezis $z$ ducha (La Genèse par l'esprit) de Juliusz Słowacki pour lequel la forme de l'arbre a été interprétée comme une forme très aboutie dans le royaume des plantes ${ }^{40}$.

A l'opposé de la langue anglaise ou française, il existe en polonais une définition à part de ce type de "forêt ». Le lexème slave puszcza qui apparaît dans le titre de la version polonaise évoque non seulement des paysages sauvages et des lieux inaccessibles, mais enrichit aussi la traduction polonaise de sens supplémentaires. Sa signification contemporaine est apparue pour la première fois au XIVe siècle et a été maintenue au XVI ${ }^{\mathrm{e}}$ siècle : le Stownik polszczyzny XVI wieku le définit en effet comme une forêt primaire et sauvage ${ }^{41}$. Au XVI $\mathrm{Xiècle}^{\mathrm{e}}$ le mot puszcza signifiait aussi un lieu esseulé et un désert. Wiesław Boryś remarque que le mot puszcza renvoyait également au " désert ", c'est à dire pustyni $a^{42}$. Cette signification était encore vivante au XIX ${ }^{e}$ siècle ; elle apparaît aussi dans le Słownik jĘzyka polskiego édité par Maurice Orgelbrand en 1861 et dans lequel sa signification est celle d'un lieu sauvage, désert : dzikie miejsce, pustynia ${ }^{43}$.

L'ancienne signification du mot puszcza résonne encore dans l'expression idiomatique de - littéralement - «la voix de celui-ci qui clame dans la forêt » (aujourd'hui «dans le désert ») głos wołajĄcego na puszczy, qui renvoie au sens de mots sans résonance auprès des autres, à l'indifférence, à des mots sans écho (słowa nieznajdujĄce oddźwį̨ku). Nous pouvons la rencontrer dans l'Uniwersalny Stownik jĘzyka polskiego de Stanisław Dubisz ${ }^{44}$ et le Stownik jĘzyka polskiego de Mieczysław Szymczak ${ }^{45}$. Le lecteur peut aussi rencontrer la version modifiée de cette expression phraséologique dans la Biblia TysiĄclecia : «la voix clamant dans le désert » (głos wołającego na pustyni). Le professeur Jan Miodek, dans son Stownik ojczyzny polszczyzny considère les deux expressions idiomatiques comme égales puisque les substantifs puszcza (la forêt) et pustynia (le désert) ont la même étymologie : pust-. En effet, puszcz ${ }^{46}$ provient de l'adjectif pustъ (vide, sans présence humaine ; pusty, bezludny $)^{47}$. Ce lien entre puszcza et le lieu vide, sans présence humaine n'apparaît pas dans le mot anglais forest ni dans le mot français forêt. Le choix du terme puszcza et non de celui de las désignant la forêt est une excellente alternative puisque l'action se déroule dans la bâtisse d'une abbaye non habitée et dans une forêt primaire. La solitude des protagonistes, à la fois réelle et spirituelle, souligne cette dimension désertique du lieu et des mots qui le décrivent. L'expression «le désert du bâtiment» (pustynia gmachu) trouvée par le traducteur et qui est récurrente dans la version polonaise du roman semble donc idéale ${ }^{48}$.

Dans cette édition, il y a aussi d'autres références à la signification double du mot puszcza : "La Motte a déjà passé plus qu'un mois dans ce désert "49. Le titre même du roman correspond aux éditions polonaises d'autres romans de Radcliffe en 1830 et 1835 Pustelnik tajemnego grobowca (L'Ermite de la tombe mystérieuse) et Pustelnik z czarnego grobowca (L'Ermite de la tombe noire). Remarquons dans ces titres la double signification du mot puszcza qui, par ailleurs, apparaît dans la forme du lexème décrit par le Stownik jĘzyka Adama Mickiewicza (Dictionnaire de la langue d'Adam Mickiewicz, 1962-1983) et est répété cinquante-huit fois dans le roman : cinquante et une fois dans la signification de forêt primaire et sauvage, sept fois dans la signification de désert ${ }^{50}$. Le mot apparait dans Konrad Wallenrod: tam góra lodu, tam puszcza go nĘci (« ici une montagne de glace, là une plaine déserte ») ${ }^{51}$ mais aussi dans Pan Tadeusz: "Forêt lituanienne, aire d'abîmes, d'antres, / Quel œil sonda jamais ton cœur jusqu'à son centre? " ${ }^{52}$. 
Pour finir, la forêt revient dans la littérature polonaise dans la prose d'Antoni Gołubiew qui a intitulé Puszcza le premier volume de son grand cycle en quatre parties Bolesław Chrobry (1947-1974), ce qui n'est pas le fruit du hasard, comme l'écrit Komendant : « Le début de la nation est lié à la forêt primaire $\aleph^{53}$ et Gołubiew marque ainsi son livre par l'exergue : « Tout a commencé dans la forêt ».

\section{Les éléments gothiques face au penzai}

Le fait que les romans gothiques se soient multipliés dans les années 1790, au moment donc de la Révolution française, faisant office de source d'inspiration pour des motifs sombres et sanglants, est particulièrement intéressant. A cette époque, outre les trois derniers romans de Radcliffe ont paru entre autres: The Monk (Le Moine) de Matthew Gregory Lewis, Montalbert (1795) de Charlotte Smith, The Mysterious Warning (Le Mystérieux Avertissement, 1796) d'Eliza Parsons, Hubert de Severac (1796) de Mary Robinson, Clermont (1798) de Regina Maria Roche, The Orphan of the Rhine (L'orphelin de Rhin, 1798) d'Eleanor Sleath, Caleb Williams (1794) et St. Leon (1799) de William Godwin.

Les éléments gothiques se sont avérés particulièrement utiles dans la création littéraire de paysages miniatures de type penzai en ces temps troubles. Cette hypothèse semble être confirmée par le marquis de Sade qui affirmait en 1800 que les œuvres de Radcliffe ont suscité un souffle de liberté, tandis que les romans gothiques en soi étaient un effet du choc révolutionnaire ressenti dans toute l'Europe ${ }^{54}$. William Hazlitt liait l'œuvre de l'auteure avec le désordre de la révolution: ses châteaux en ruines reflétaient les structures chancelantes des anciens temps ${ }^{55}$. Les éléments gothiques s'avéraient particulièrement adéquats pour créer un petit panorama de cette époque. En 1791, au moment de la parution de The Romance of the Forest, la constitution avait été adoptée en France, tandis qu'en 1794, lorsque commença la traduction du roman, la révolution battait son plein et récoltait ses plus grands succès. Une année plus tôt le roi Louis XVI et la reine Marie-Antoinette avaient été guillotinés.

Dans The Romance of the Forest, injustement considéré par Zofia Sinko comme le moins terrifiant de tous les ouvrages de Radcliffe ${ }^{56}$, les éléments caractéristiques propres au roman gothique sont bien présents. Un grand rôle important est joué par l'architecture qui acquiert le statut de héros à part entière. La petitesse de l'homme, son caractère disparate sont soulignés par le mystère du lieu et sa démesure qui, dans le monde des souterrains, des couloirs, des labyrinthes remplis de toiles d'araignées, le projette dans la dimension du microcosme de l'abbaye :

La nature (vent, pluie, animaux) déjà évoquée auparavant est renforcée par toute une panoplie d'objets qui sont autant d'éléments propres au roman gothique: le squelette retrouvé dans le coffre, le manuscrit mystérieux, le couteau rouillé, le fantôme, les bijoux cachés dans un tombeau reculé dans la forêt, le crime, les duels, les enlèvements, la vengeance, le danger du viol, le suicide. Adeline, l'héroïne principale, est une vierge innocente, persécutée par un marquis au caractère de véritable voyeur. Pourtant, après une lecture attentive et une analyse appuyée, nous nous rendons compte qu'il est difficile de situer réellement le caractère des personnages entre le côté obscur et le comportement juste et droit. Les personnages de Pierre de La Motte, de sa femme et de son serviteur en témoignent en tant qu'exemples difficilement qualifiables du point de vue d'une moralité irréprochable. 


\section{Les diverses façons de mener une narration}

Dans son roman, Radcliffe mène la narration dans un mode caractéristique et recherché. Il s'avère que l'utilisation du bagage scientifique et des acquis des formalistes russes sont utiles pour leur analyse. A la place de l'action aristotélicienne (praxis - $\pi \rho \alpha \xi \zeta)$ et du fil rouge de l'histoire (mythos - $\mu$ v́⿴囗o)), ils introduisent de nouveaux concepts tels que fabula (ФАБУЛА) et sûžet (СЮЖЕТ). Fabula signifiait pour eux une histoire prête que l'auteur utilisait dans son œuvre. Tandis que sûžet renvoyait à la façon de raconter l'histoire. L'histoire même de l'histoire. Dans The Romance of the Forest, nous pouvons déceler la tension qui ressort d'entre ces deux catégories. Elle relève du fait que Radcliffe ne présente pas une histoire soumise à un ordre chronologique, de la naissance d'Adeline au jugement à Paris : au contraire, au moment des premiers mots du roman, l'annonce du meurtre du père d'Adeline a déjà eu lieu. Nous pouvons deviner ce qui arrivera, en le déduisant des propos des protagonistes, de leurs comportements et de leurs décisions. Le contenu du roman est un dévoilement graduel des évènements du passé et le lecteur a pour tâche de reconstruire par lui-même tout ce microcosme, le fil de l'histoire ainsi que les lois qui y règnent.

Ce n'est que dans les dernières pages de l'œuvre que le lecteur peut confirmer ses hypothèses de reconstruction de l'histoire et apprécier pleinement l'ambiance mystérieuse - ce que permet le sûžet. Cette façon d'écrire a d'ailleurs été utilisée par les auteurs de romans policiers tels qu'Agatha Christie (1890-1976) afin d'augmenter le suspense.

L'autre moyen repose sur le fait de passer d'une manière fluide entre la narration personnelle à la narration auctoriale : d'une part nous savons seulement très peu de ce que sait Adeline, d'autre part nous avons la perspective plus large de quelqu'un qui regarde d'un point de vue plus grand. Cette démarche augmente considérablement la dimension de peur enfuie et donne de l'ampleur à ce petit monde encerclé dans ses propres sentiments et intrigues.

\section{Une construction minutieuse et unique}

Radcliffe a réussi à enfermer dans le monde littéraire une miniature d'un monde en autarcie - un monde autosuffisant. Ses contemporains traitaient cet ouvrage comme représentant plus qu'un ouvrage destiné à au divertissement. Joanna Southcott, prophétesse se considérant comme l'élue de Dieu, en est un parfait exemple. Tout a commencé en 1803, soit six ans après avoir commencé à recevoir des messages de « La Petite Voix Calme ». Le 13 août 1803 naît l'analyse divine de The Romance of the Forest, composée de vingt-huit lignes en prose et vingt-huit distiques rimés, dictés, selon les dires de la prophétesse, par l'Esprit de Dieu même. Selon lui, le roman de Radcliffe a été savamment inventé et imprimé. Son but était de montrer le véritable visage du crime et la façon dont il peut subsister longtemps sans être révélé.

La fable même du livre contient en soi une miniature du monde qui lui est contemporain, une allégorie du tourment des semblables de Joanna Southcott et de leur prophétie concernant les desseins divins. Selon l'Esprit, Philippe de Montalt est l'équivalent de Satan, et les sbires dont il loue les services sont les acolytes des forces du mal dont la 
présence dans le monde est traduite par le fait que l'on se moque des prophéties de la visionnaire, identifiée à Adeline. Le mystère soufflant sur l'identité du père de l'hérö̈ne principale répond au choix que doit effectuer Joanna entre l'acceptation des conventions adoptées par la société ou la voix de son véritable Père. De même que Montalt a été condamné pour le meurtre du père d'Adeline, de même, selon l'Esprit, un procès sera fait à Satan. C'est alors que Joanna Southcott et ses adeptes triompheront. Cette certitude est devenue très populaire parmi le courant prophétique. Le troisième roman de l'auteure était donc avec certitude, aux yeux de Southcott et de ses 10000 adeptes, une œuvre divine ${ }^{58}$.

Ce n'est donc peut-être pas un hasard si The Romance of the Forest a été traduit en français en 1794, alors que durait la Révolution française. Il est très probable que les révolutionnaires se soient identifiés au roman : l'action se déroule d'ailleurs justement en France. L'œuvre contiendrait ainsi une miniature de la situation politique française. Le sort d'Adeline pouvait nourrir leur espoir de victoire qui marquerait la fin de l'injustice et le recouvrement des droits des individus opprimés, telle Adeline dans The Romance of the Forest. Les Polonais ont pu quant à eux être fascines par l'interprétation française du roman, eux qui voyaient également une miniature de la situation politique de leur pays et des rapports avec leurs envahisseurs. Il n'est pas exclu que la décision de traduire le roman ait été prise lorsque Mickiewicz a publié une œuvre étonnamment similaire dans sa signification et dans ses motifs.

Les différents caractères littéraires donnent leur valeur au roman de Radcliffe: une construction ordonnée à l'image de la composition penzai, où il n'y a pas de place pour le hasard. En même temps, son apparentement à la cathédrale gothique reste valable en tant qu'image miniaturisée puisqu'elle veut nous projeter dans la comparaison avec le cosmos. Le moindre détail devient symbolique et signifiant. Ainsi tous les motifs, chiffres et accessoires dans The Romance of the Forest se caractérisent par une signification précise et importante.

Rappelons que les cathédrales gothiques et le penzai ont ce trait en commun: il est impossible d'en faire une copie exacte, ni au niveau de la forme, ni au niveau de la matière, ni au niveau des couleurs. Si nous comparons donc l'œuvre littéraire à un genre de penzai - sa version polonaise dans ce cas-ci, indépendamment de l'original (et de la version française) devient à leur tour un genre de penzai littéraire, notamment à cause des différents bagages linguistiques.

L'auteur de la version polonaise de The Romance of the Forest a introduit dans le texte du roman des significations nouvelles en utilisant par exemple le terme puszcza au lieu de las. Ainsi, les miniatures des paysages sont aussi créées par la volonté de rendre la poétique de toute la composition de minuscules détails dans un monde bien défini qui permet de ressentir de grandes émotions et ouvre la voie au fantastique, aux mondes minuscules ou grands des futurs romans polonais des $\mathrm{XX}^{\mathrm{e}}$ et $\mathrm{XXI}^{\mathrm{e}}$ siècles. 


\section{NOTES}

1. Zagoskine M.N., Iskusitel (Le Tentateur), [online], http://az.lib.ru/z/zagoskin_m_n/ text_0040.shtml, consulté le 18 mai 2015.

2. Duras M., Écrire, Gallimard, Paris, 1993, p. 17.

3. Voir. Arnaud P., « Note sur l'édition », in: Radcliffe A., Les Mystères de la forêt, traduit par François Soulès, éd. Pierre Arnaud, Gallimard, Paris, 2011, p. 55. Nous utiliserons cette édition. Les prochaines mentions seront notées [Mystères], suivi du numéro de page (chiffres latins).

4. Radcliffe A., La forêt, ou l'Abbaye de Saint-Clair, traduit par François Soulès, Denné, Paris, 1794.

5. Dans cet article, nous utilisons la version polonaise du roman tout en consultant l'original et sa traduction française.

6. Voir : «Święty obszar i sakralizacja świata » (L'espace sacré et la sacralisation du monde),in : Antropologia kultury (Anthropologie culturelle), sous la direction de GodlewskiG. et alii, Wydawnictwo Uniwersytetu Warszawskiego, Varsovie, 2005, p. 155.

7. «Opactwo» (Abbaye), in: Kopaliński W., Słownik wyrazów obcych i zwrotów grzecznościowych (Dictionnaire des mots d'origine étrangère et des formules de politesse), Wiedza Powszechna, Varsovie, 1985, p. 303.

8. Radcliffe A., Puszcza czyli opactwo St.Clair (La forêt, ou l'Abbaye de Saint-Clair), t. IV, Imprimerie Józef WĘcki, Varsovie, 1829, pp. 187-188. Les prochaines références seront notées [ Puszcza], suivi du numéro de tome (chiffres romains) et de page (chiffres latins).

9. «Teodor» (Théodore), in: Grzenia J., Nasze imiona (Nos prénoms), Wydawnictwo Naukowe PWN, Varsovie, 2002, p. 307.

10. Puszcza, I, 54 .

11. «Trzy » (Trois), in : Lurker M., Stownik obrazów i symboli biblijnych (Dictionnaire des images et des symboles bibliques), traduit par K. Romaniuk, Pallottinum, Poznań, 1989, p. 252.

12. «Paw» (Paon), in: Tresidder J., Stownik symboli (Dictionnaire des symboles), traduit par Bożenna Stokłosa, Wydawnictwo RM, Varsovie, 2001, p. 158.

13. «Paw» (Paon), in : Kopaliński W., Słownik mitów i tradycji kultury (Dictionnaire des mythes et des traditions culturelles), Państwowy Instytut Wydawniczy, Varsovie, 1985, p. 843.

14. « Koło » (Roue), in : Lurker M., op. cit., pp. 89-90.

15. « Korzeń » (Racine), in : ibid., p. 93.

16. « Wiatr » (Vent), in : ibid., p. 259.

17. Idem.

18. « Noc» (Nuit), in : ibid., p. 136.

19. Tomkowski J., "Słowa i sny» (Les mots et les rêves), in : Pismo Literacko-Artystyczne (Revue littéraire et artistique), t. I, Cracovie, 1989, p. 60.

20. «Błyskawice i grzmoty » (Éclairs et tonnerres), in : Lurker M., op. cit. p. 26.

21. Puszcza, I, 203-204; Mystères, 153.

22. Puszcza, I, 172 ; Mystères, 140.

23. Puszcza, I, 61 ; Mystères, 90.

24. Puszcza, IV, 142 ; Mystères, 474.

25. Puszcza, I, 61 ; Mystères, 90.

26. Puszcza, I, 17 (Niebo ciebie na mój ratunek zesłało); Mystères, 70.

27. Puszcza, IV, 188 (zdaje nam siĘ, że jej ocalenie jest dziełem mĄdrości wyższej nad ludzkĄ); Mystères, 496. 
28. Puszcza, IV, 195 (okoliczności, które towarzyszyły odkryciu tych papierów nie zdawały jej się być skutkiem przypadku, ale raczej zrzĄdzeniem władzy nadnaturalnej) ; Mystères, 499.

29. Des tentatives similaires de déchiffrement des sens cachés dans les textes litteraire a l'aide de codes chiffres ont ete réalisés par des chercheurs renommés comme Juliusz Kleiner (1886-1957) et Wiktor Weintraub (1908-1988). Voir : J. Kleiner, « 40 i 4 czy 44 » (40 et 4 ou 44), in : Ruch Literacki (Le mouvement littéraire), t. II, Cracovie, 1930, pp.35-44 et WeintraubW., «Geometria w funkcji chwytu literackiego i Mickiewiczowskiego czterdzieści i cztery» (La géométrie dans la fonction du motif littéraire et mickiewiczien quarante-quatre), in : PamiĘtnik Literacki (Journal littéraire), t. IV, Cracovie, 1980, pp. 287-295.

30. Edgeworth M., Chosen Letters (Lettres choisies), J. Cape, Londres, 1931, p. 58. Cité d'après : Norton R., Mistressof Udolpho: The Life of Radcliffe (La dame d'Udolphe: la vie de Radcliffe), Leicester University Press, Londres, 1999,p. 89.

31. Voir Kiełczewski B. \& Wiśniewski J., Kulturotwórcza rola lasu (Le rôle créatif de la forêt dans la culture), Ośrodek Kultury Leśnej w Gołuchowie, Gołuchów, 2012.

32. Norton R., op. cit., p. 88.

33. Lurker M., Przesłanie symboli w mitach, kulturach i regionach (Le message des symboles dans les mythes, les cultures et les régions), Znak, Cracovie, 1994, p. 66.

34. Norton R., op. cit., p. 88.

35. Puszcza, I, 47 (Adelina dotĄd spokojna wykrzyknĘła głosem podziwienia i bojaźni. Jakiś rodzaj zdumienia opanował jej duszE,, i sprawił drżenie jej serca); Mystères, 84.

36. Hussowski M., Mikołaja Hussowskiego pieśń o żubrze jego postaci, dzikości i o polowaniu na niego (Les personnages, la vie sauvage et la chasse au bison. Le chant sur le bison de Mikołaj Hussowski), traduction de J.Kasprowicz, Białowieski Park Narodowy - Dział Wydawnictw, Białowieża, 1994.

37. Komendant T., « Domowe drzewa: las w literaturze », (Nos arbres domestiques : la forêt dans la littérature), in : $W$ głąb lasu: las $w$ polskiej kulturze $i$ sztuce (Au plus profond de la forêt : la forêt dans la culture et la littérature polonaises), Wydawnictwo Sport i Turystyka, Varsovie, 1982, p. 148.

38. Sinko Z., Proza fabularna $w$ czasopismach polskich 1801-1830 (La prose fictionnelle dans les revues polonaises de 1801 à 1830), Ossolineum, Wrocław, 1988, p. 122.

39. Komendant T., op. cit., p. 148.

40. Voir. Grzęda E., Funkcja i estetyka motywów drzewa i lasu w twórczości Juliusza Słowackiego (La fonction et l'esthétique des motifs des arbres et de la forêt dans les ouvrages de Juliusz Słowacki),Oficyna Wydawnicza Oddziału Wrocławskiego PTTK Sudety, Wrocław, 2000, p. 185.

41. « Pierwotny dziki las» : «Puszcza » (Forêt), in : Słownik polszczyzny XVI wieku (Dictionnaire de la langue polonaise du XVI ${ }^{\mathrm{e}}$ siècle), sous la direction de Mayenowa M. R., t. XXXIV, Wydawnictwo Instytutu Badań Literackich PAN, Varsovie, 2010, p. 485.

42. «Puszcza» (Forêt), in : Boryś W., Słownik etymologiczny jદ̨zyka polskiego (Dictionnaire étymologique de la langue polonaise), Wydawnictwo Literackie, Cracovie, 2005, p. 505.

43. «Puszcza » (Forêt), in : Słownik jŁ̨zyka polskiego (Dictionnaire de la langue polonaise), sous la direction de Czepieliński et alii, t. II, Maurycy Orgelbrand, Vilnius, 1861, p. 1331.

44. «Głos» (Voix), in : Uniwersalny słownik jĘzyka polskiego (Dictionnaire universel de la langue polonaise), sous la direction de Dubisz S., t. I, Wydawnictwo Naukowe PWN, Varsovie, 2003, p. 1021.

45. «Puszcza» (Forêt), in : Słownik jĘzyka polskiego (Dictionnaire de la langue polonaise), M. Szymczak (dir.), t. II, op. cit., s. 1082.

46. «Głos » (Voix), in : Miodek J., Słownik ojczyzny polszczyzny (Dictionnaire de la langue polonaise comme langue maternelle), sous la direction de Zaśko-Zielińska M. \& Piekot T., Wydawnictwo Europa, Wrocław, 2002, p. 222. 
47. «Puszcza » (Forêt), in : Boryś W., op. cit., p. 505.

48. Puszcza, I, 41.

49. Puszcza, I, 127. « La Motte już wiĘcej jak jeden miesiĄc przepĘdził w téj pustyni. »

50. «Puszcza » (Forêt), in : Słownik jĘzyka Adama Mickiewicza (Dictionnaire de la langue d'Adam Mickiewicz), sous la direction de Górski K. et Hrabec S., t. VII, Ossolineum, Wrocław, 1971, pp. 278-279.

51. Mickiewicz A., «Konrad Wallenrod» (Conrad Wallenrod), in : Powieści poetyckie (Romans poétiques), Czytelnik, Varsovie, 1983, p. 131. Version française : Mickiewicz A., Conrad Wallenrod: légende historique d'après les chroniques de Lithuanie et de Prusse, trad. de l'un des fils de l'aut., Paris, 1886, p. 68.

52. Mickiewicz A., Pan Tadeusz (Messire Thadée), sous la direction de Pigoń S., Ossolineum, Wrocław, 2012, p. 213 (Któż zbadał puszcz litewskich przepastne krainy / Aż do samego środka, do jądra gĘstwiny?). Version française: Mickiewicz A., Pan Tadeusz ou La dernière expédition judiciaire en Lituanie, traduction de R. Bourgeois, Les Éditions Noir sur Blanc, Montricher, 2005, p. 135.

53. Komendant T., op. cit., p. 148.

54. Norton R., op. cit., p. 166.

55. Hazlitt W, Lectures on the English Comic Writers (Cours sur les écrivains anglais comiques), Taylor and Hessey, London, 1819, p. 244.

56. Sinko Z., Powieść angielska osiemnastego wieku a powieść polska lat 1764-1830 (Le roman anglais du XVIII ${ }^{e}$ siècle et le roman polonais dans les années 1764-1830), Państwowy Instytut Wydawniczy, Varsovie, 1961, p. 142. Nous pensons au contraire que ce livre est plutôt le plus effrayant (NdlA).

57. Mystères, 81-82 ; Puszcza, I, 40 : « Zbliża się, i widzi szczĄtki gotyckie opactwa : postawione było na pewnej wyniosłości, i otoczone wysokimi i g̨̨stymi drzewami, które zdawały się być współczesne z budowlą, i rzucały na około jakiś cień romantyczny. Większa czĘść budowli była w rozwaliskach, a to co sį̨ czasowi oparło, przedstawiało jeszcze okropniejszy widok przez zbyt dawnĄ i zaniedbanĄ postawĘ. Ogromne urwiska wieży wschodniej, prawie całkowicie zniszczonej leżały rozrzucone wśród wysokiej trawy, z którĄ wolno igrały powiewy zefiru ».

58. Norton R., op. cit., pp. 90-91.

\section{AUTHORS}

\section{ANNA JAWORSKA}

Université de Varsovie 